"Mircea cel Batran" Naval Academy Scientific Bulletin, Volume XIX - 2016 - Issue 2

The journal is indexed in: PROQUEST / DOAJ / Crossref / EBSCOhost / INDEX COPERNICUS / DRJI / OAJI I

JOURNAL INDEX I I2OR / SCIENCE LIBRARY INDEX / Google Scholar / Academic Keys/ ROAD Open Access I

Academic Resources / Scientific Indexing Services / SCIPIO / JIFACTOR

\title{
STUDIES REGARDING THE STRUCTURAL RESPONSE OF A 2000X100X4MM PLATE AFTER THE IMPACT WITH A 6.2KG CYLINDRICAL BODY CONSIDERING THE EQUIVALENT STRESS
}

\author{
Marian RISTEA ${ }^{1}$ \\ Daniel MARASESCU ${ }^{2}$ \\ Adrian POPA ${ }^{3}$ \\ Ionut-Cristian SCURTU ${ }^{4}$
}

${ }^{1}$ Assist prof. PhD Eng. Marine Engineering and Naval Weapons Department
2 PhD attendee, Marine Engineering and Naval Weapons Department
${ }^{3}$ Assist. prof. PhD. Eng., Marine Engineering and Naval Weapons Department
${ }^{4}$ Principal Instructor, PhD Eng. "Mircea cel Batran" Naval Academy

Abstract: The present article is included in a wider series of studies which were developed by the development team; the initial premises of the article consists in an impact analysis of a $6.2 \mathrm{~kg}$ cylindrical body

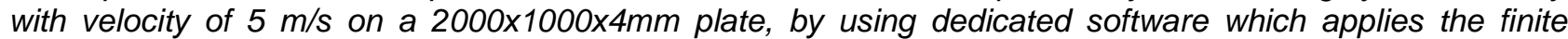
element theory.

Keywords: vonMises stress, impact body, energy impact, distortion.

This paperwork belongs to a series of papers covering a complex study regarding the impact of a $6.2 \mathrm{~kg}$ cylindrical body on a $2000 \times 1000 \times 4 \mathrm{~mm}$ plate.

This study is considering the plate to be fixed on all 4 sides and a cylindrical body hits it with impact $5 \mathrm{~m} / \mathrm{s}$ impact speed.

Also, the standard earth gravity is considered to be active.

The studies were carried out in ANSYS 12.1.

Both, plate and body are considered to be made from structural steel.

At the impact, the geometry is presented in below figure:

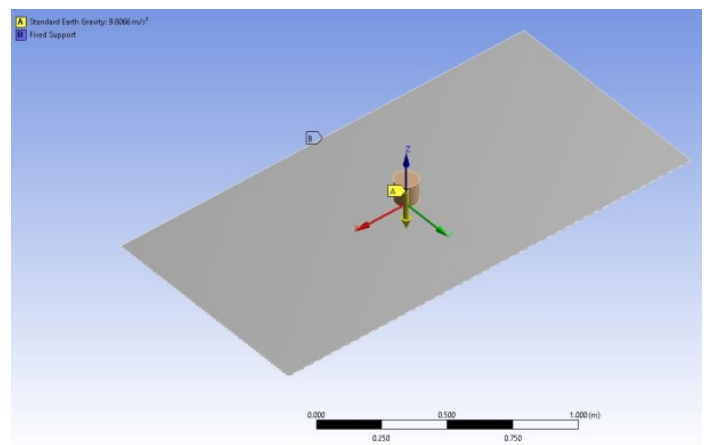

Figure 1 The geometry at the impact

Also, in Figure 1are presented the boundary conditions and the axis of coordinate. $X$ is the red vector, $Y$ is the green vector and $Z$ is the blue vector.

The origin is situated in the middle of the plate, at the intersection of the median surfaces.

DOI: 10.21279/1454-864X-16-12-044

(c) 2015. This work is licensed under the Creative Commons Attribution-Noncommercial-Share Alike 4.0 License.

The two bodies were meshed as below:

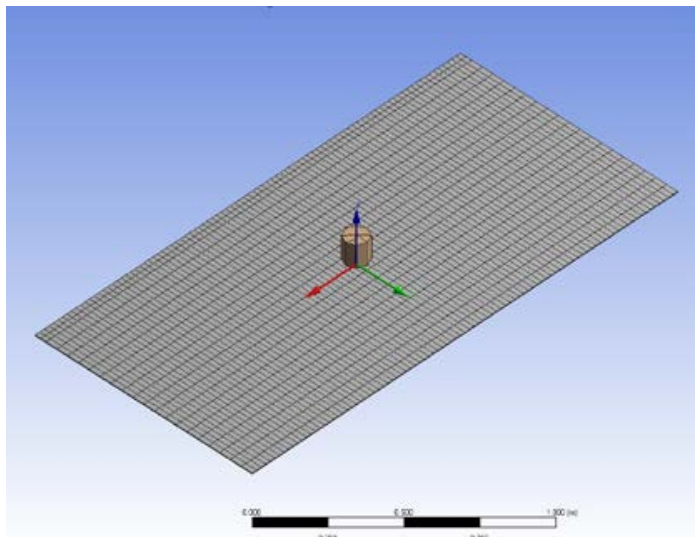

Figure 2 The meshed structure

The mesh of the plate consists in 4200 nodes and 1980 elements.

The simulations were a dynamic one, having the end time of 0.3 seconds.

At the beginning of the simulation, the hammer is situated at $20 \mathrm{~mm}$ above the plate.

The analysis was carried out in 80 steps, from 1 to 81 , equally distributed.

The steps distributed on time are presented below:

Table 1 Steps distribution on time

\begin{tabular}{|r|l|r|l|}
\hline Step & Time [s] & Step & Time [s] \\
\hline 1 & $1.18 \mathrm{E}-38$ & 21 & $7.50 \mathrm{E}-02$ \\
\hline 2 & $3.75 \mathrm{E}-03$ & 22 & $7.88 \mathrm{E}-02$ \\
\hline 3 & $7.50 \mathrm{E}-03$ & 23 & $8.25 \mathrm{E}-02$ \\
\hline 4 & $1.13 \mathrm{E}-02$ & 24 & $8.63 \mathrm{E}-02$ \\
\hline
\end{tabular}


"Mircea cel Batran" Naval Academy Scientific Bulletin, Volume XIX - 2016 - Issue 2

The journal is indexed in: PROQUEST I DOAJ / Crossref / EBSCOhost / INDEX COPERNICUS / DRJI / OAJI I JOURNAL INDEX I I2OR / SCIENCE LIBRARY INDEX / Google Scholar / Academic Keys/ ROAD Open Access I Academic Resources / Scientific Indexing Services / SCIPIO / JIFACTOR

\begin{tabular}{|r|l|r|r|}
\hline Step & Time [s] & Step & Time [s] \\
\hline 5 & $1.50 \mathrm{E}-02$ & 25 & $9.00 \mathrm{E}-02$ \\
\hline 6 & $1.88 \mathrm{E}-02$ & 26 & $9.38 \mathrm{E}-02$ \\
\hline 7 & $2.25 \mathrm{E}-02$ & 27 & $9.75 \mathrm{E}-02$ \\
\hline 8 & $2.63 \mathrm{E}-02$ & 28 & 0.10125 \\
\hline 9 & $3.00 \mathrm{E}-02$ & 29 & 0.105 \\
\hline 10 & $3.38 \mathrm{E}-02$ & 30 & 0.10875 \\
\hline 11 & $3.75 \mathrm{E}-02$ & 31 & 0.1125 \\
\hline 12 & $4.13 \mathrm{E}-02$ & 32 & 0.11625 \\
\hline 13 & $4.50 \mathrm{E}-02$ & 33 & 0.12 \\
\hline 14 & $4.88 \mathrm{E}-02$ & 34 & 0.12375 \\
\hline 15 & $5.25 \mathrm{E}-02$ & 35 & 0.1275 \\
\hline 16 & $5.63 \mathrm{E}-02$ & 36 & 0.13125 \\
\hline 17 & $6.00 \mathrm{E}-02$ & 37 & 0.135 \\
\hline 18 & $6.38 \mathrm{E}-02$ & 38 & 0.13875 \\
\hline 19 & $6.75 \mathrm{E}-02$ & 39 & 0.1425 \\
\hline 20 & $7.13 \mathrm{E}-02$ & 40 & 0.14625 \\
\hline
\end{tabular}

\begin{tabular}{|r|r|r|r|}
\hline Step & Time [s] & Step & Time [s] \\
\hline 41 & 0.15 & 61 & 0.225 \\
\hline 42 & 0.15375 & 62 & 0.22875 \\
\hline 43 & 0.1575 & 63 & 0.2325 \\
\hline 44 & 0.16125 & 64 & 0.23625 \\
\hline 45 & 0.165 & 65 & 0.24 \\
\hline 46 & 0.16875 & 66 & 0.24375 \\
\hline 47 & 0.1725 & 67 & 0.2475 \\
\hline 48 & 0.17625 & 68 & 0.25125 \\
\hline 49 & 0.18 & 69 & 0.255 \\
\hline 50 & 0.18375 & 70 & 0.25875 \\
\hline 51 & 0.1875 & 71 & 0.2625 \\
\hline 52 & 0.19125 & 72 & 0.26625 \\
\hline 53 & 0.195 & 73 & 0.27 \\
\hline 54 & 0.19875 & 74 & 0.27375 \\
\hline 55 & 0.2025 & 75 & 0.2775 \\
\hline 56 & 0.20625 & 76 & 0.28125 \\
\hline 57 & 0.21 & 77 & 0.285 \\
\hline 58 & 0.21375 & 78 & 0.28875 \\
\hline 59 & 0.2175 & 79 & 0.2925 \\
\hline 60 & 0.22125 & 80 & 0.29625 \\
\hline & & 81 & 0.3 \\
\hline & & & \\
& &
\end{tabular}

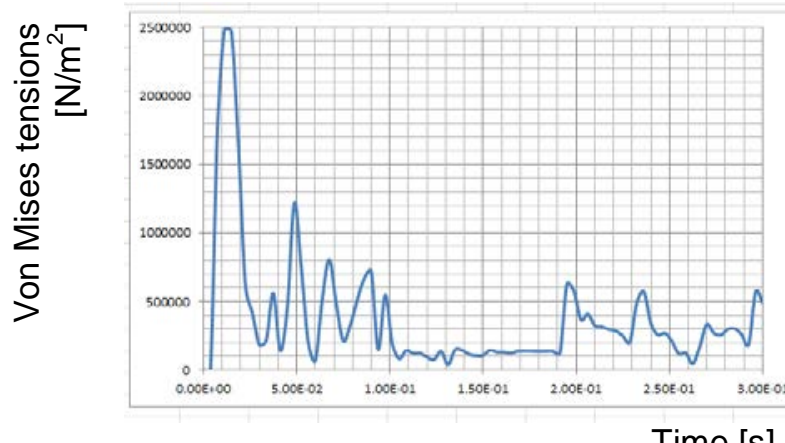

Figure 3 Time evolution of the minimum von Mises stress

The time evolution of the maximum values of the von Mises tension is presented in Figure 4

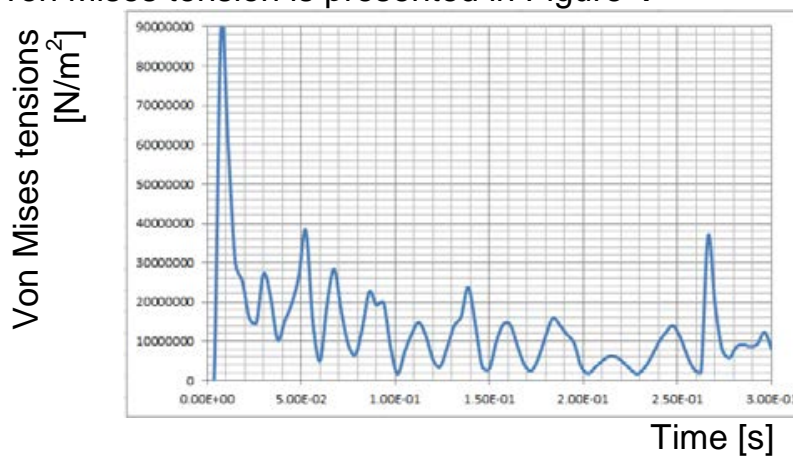

Figure 4 Time evolution of the maximum von Mises stress

Von Mises distribution diagrams are presented below:

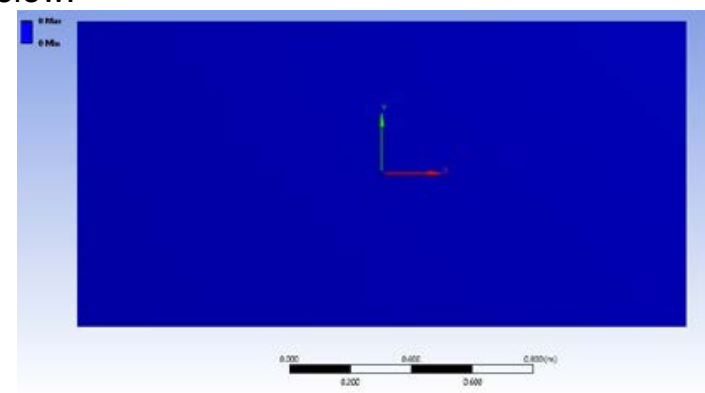

Figure 5 Equivalent stress (Von Mises) distribution diagram for step 1

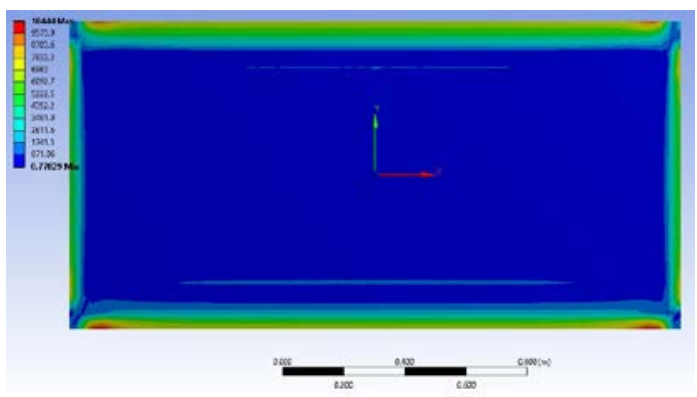

Figure 6 Equivalent stress (Von Mises) distribution diagram for step 2

The time evolution of the minimum values of the von Mises tension is presented in Figure $\mathbf{3}$ 
"Mircea cel Batran" Naval Academy Scientific Bulletin, Volume XIX - 2016 - Issue 2

The journal is indexed in: PROQUEST I DOAJ / Crossref / EBSCOhost / INDEX COPERNICUS / DRJI / OAJI I JOURNAL INDEX / I2OR / SCIENCE LIBRARY INDEX / Google Scholar / Academic Keys/ ROAD Open Access I Academic Resources / Scientific Indexing Services / SCIPIO / JIFACTOR

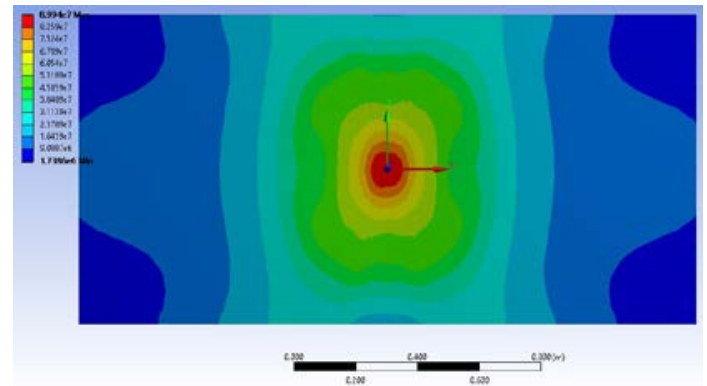

Figure 7 Equivalent stress (Von Mises) distribution diagram for step 3

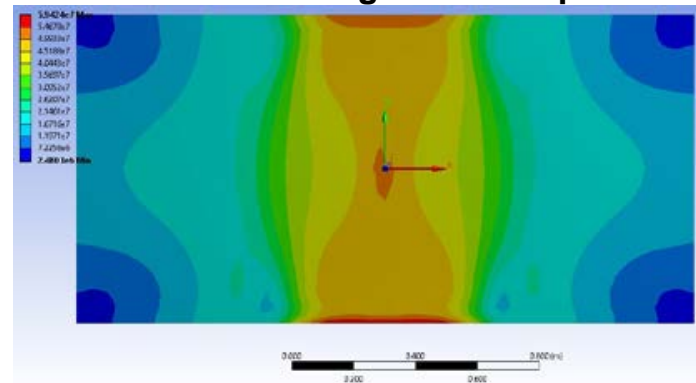

Figure 8 Equivalent stress (Von Mises) distribution diagram for step 4

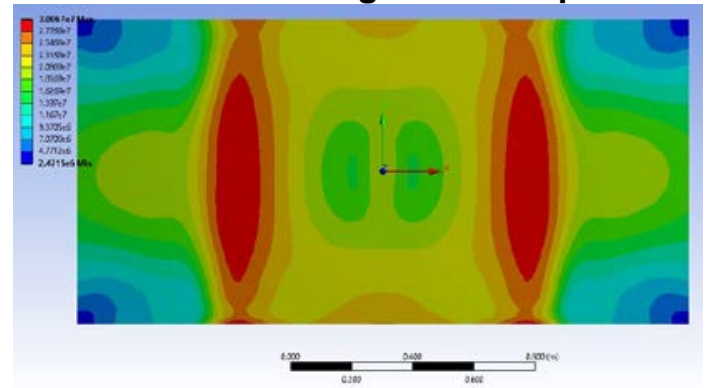

Figure 9 Equivalent stress (Von Mises) distribution diagram for step 5

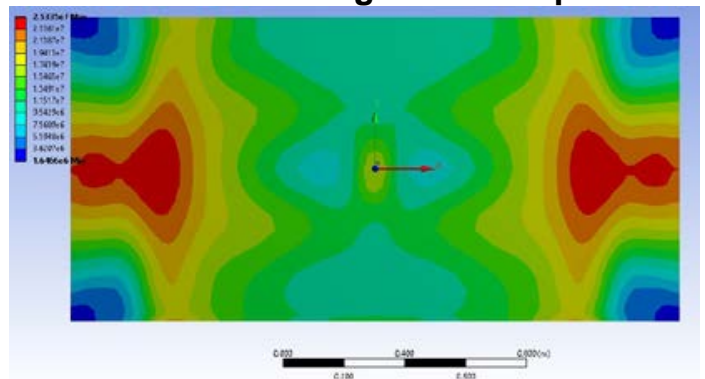

Figure 10 Equivalent stress (Von Mises) distribution diagram for step 6

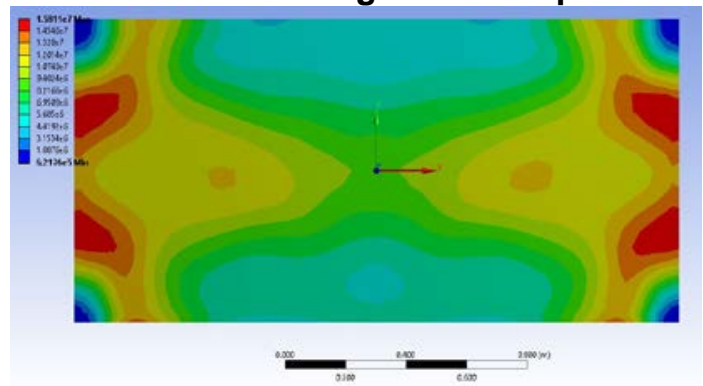

Figure 11 Equivalent stress (Von Mises) distribution diagram for step 7

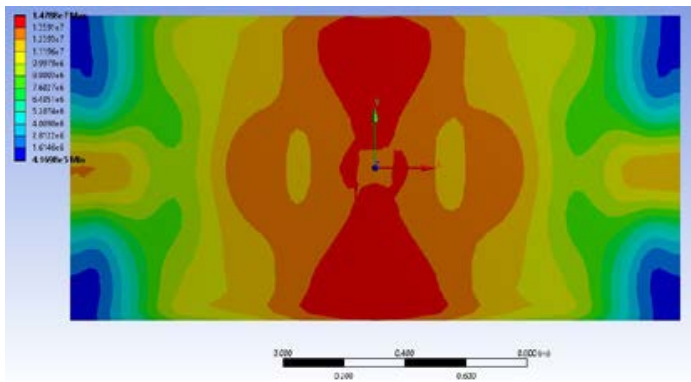

Figure 12 Equivalent stress (Von Mises) distribution diagram for step 8

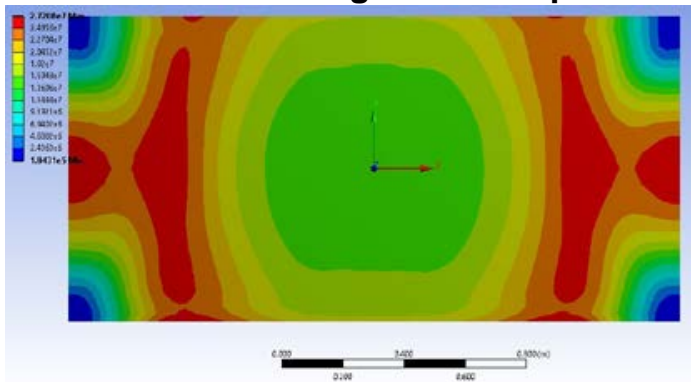

Figure 13 Equivalent stress (Von Mises) distribution diagram for step 9

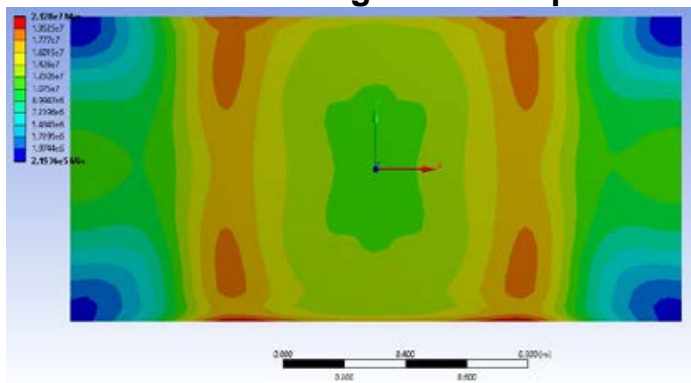

Figure 14 Equivalent stress (Von Mises) distribution diagram for step 10

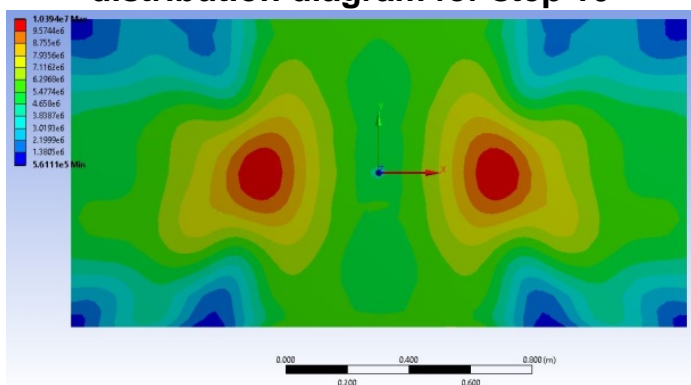

Figure 15 Equivalent stress (Von Mises) distribution diagram for step 11

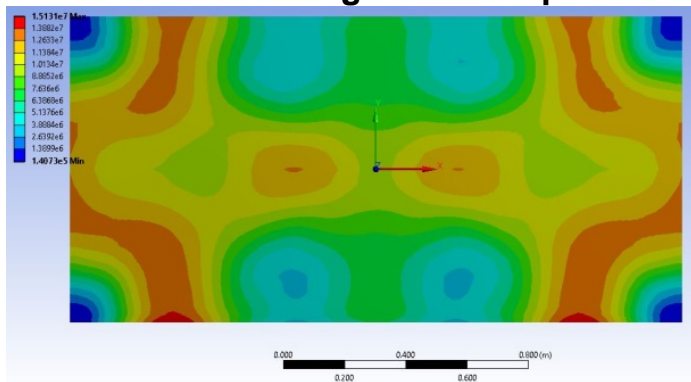

Figure 16 Equivalent stress (Von Mises) distribution diagram for step 12 
"Mircea cel Batran" Naval Academy Scientific Bulletin, Volume XIX - 2016 - Issue 2 The journal is indexed in: PROQUEST I DOAJ / Crossref / EBSCOhost / INDEX COPERNICUS / DRJI / OAJI I JOURNAL INDEX I I2OR / SCIENCE LIBRARY INDEX / Google Scholar / Academic Keys/ ROAD Open Access I Academic Resources / Scientific Indexing Services / SCIPIO / JIFACTOR

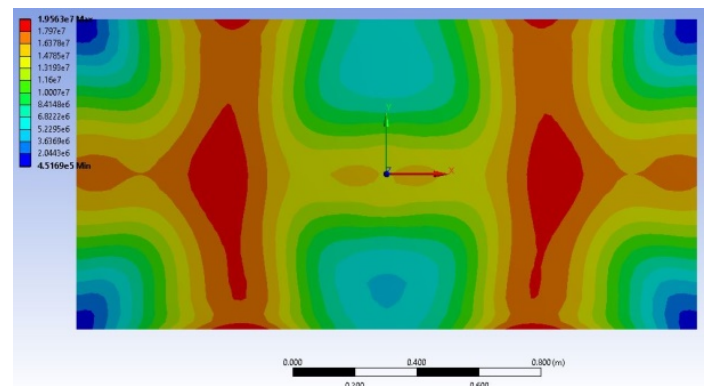

Figure 17 Equivalent stress (Von Mises) distribution diagram for step 13

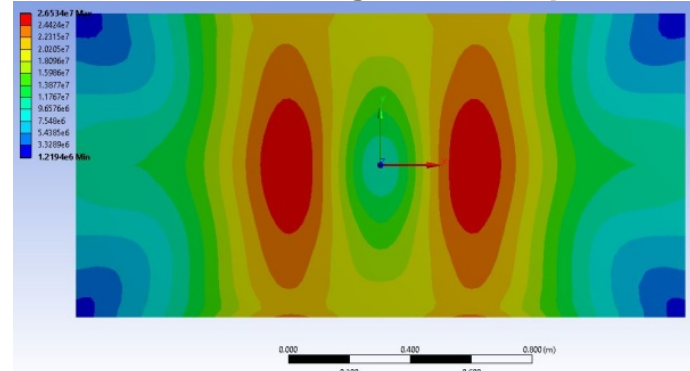

Figure 18 Equivalent stress (Von Mises) distribution diagram for step 14

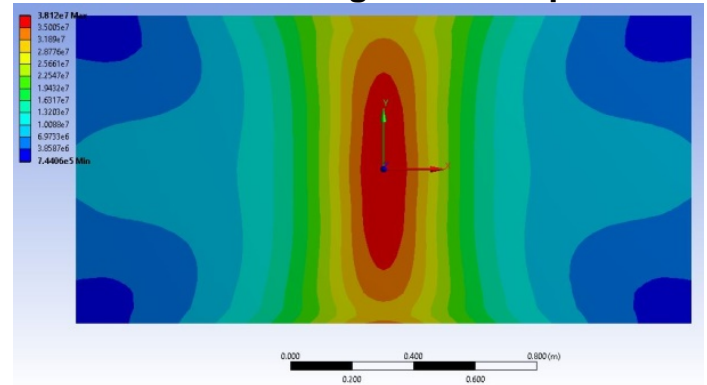

Figure 19 Equivalent stress (Von Mises) distribution diagram for step 15

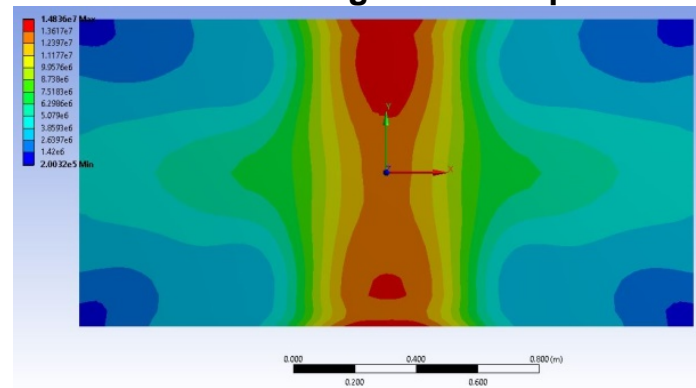

Figure 20 Equivalent stress (Von Mises) distribution diagram for step 16

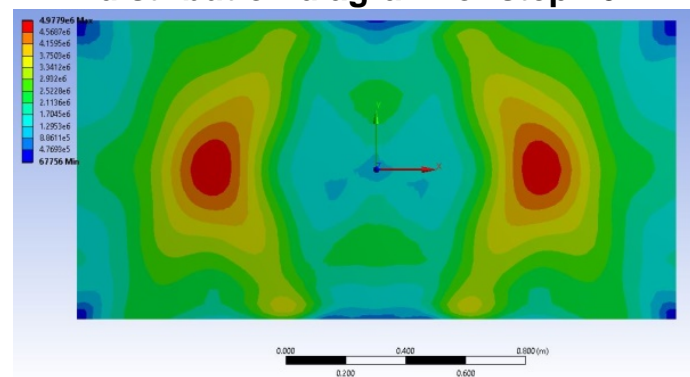

Figure 21 Equivalent stress (Von Mises) distribution diagram for step 17

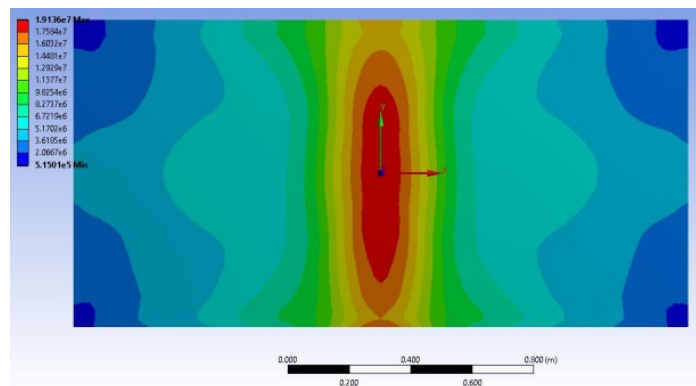

Figure 22 Equivalent stress (Von Mises) distribution diagram for step 18

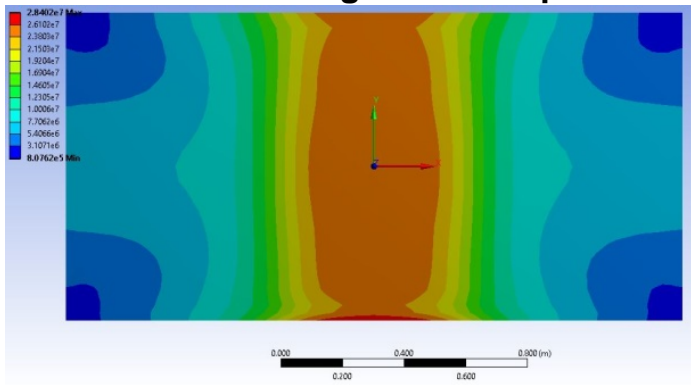

Figure 23 Equivalent stress (Von Mises) distribution diagram for step 19

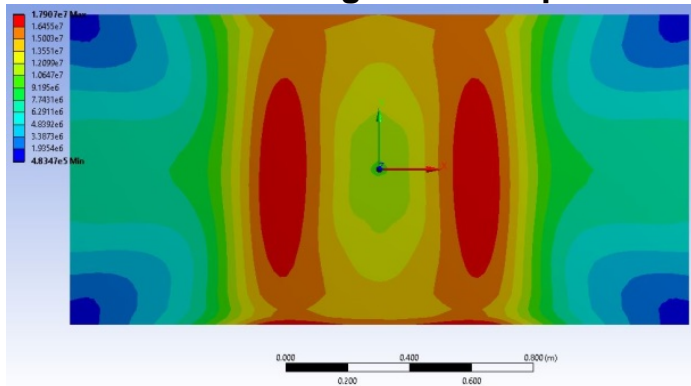

Figure 24 Equivalent stress (Von Mises) distribution diagram for step 20

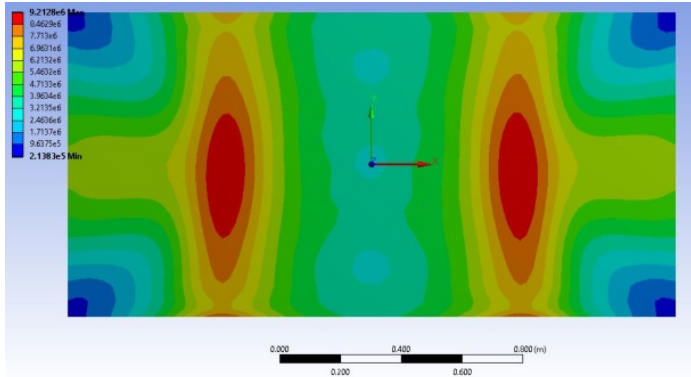

Figure 25 Equivalent stress (Von Mises) distribution diagram for step 21 
"Mircea cel Batran" Naval Academy Scientific Bulletin, Volume XIX - 2016 - Issue 2 The journal is indexed in: PROQUEST I DOAJ / Crossref / EBSCOhost / INDEX COPERNICUS / DRJI / OAJI I JOURNAL INDEX I I2OR / SCIENCE LIBRARY INDEX / Google Scholar / Academic Keys/ ROAD Open Access I Academic Resources / Scientific Indexing Services / SCIPIO / JIFACTOR

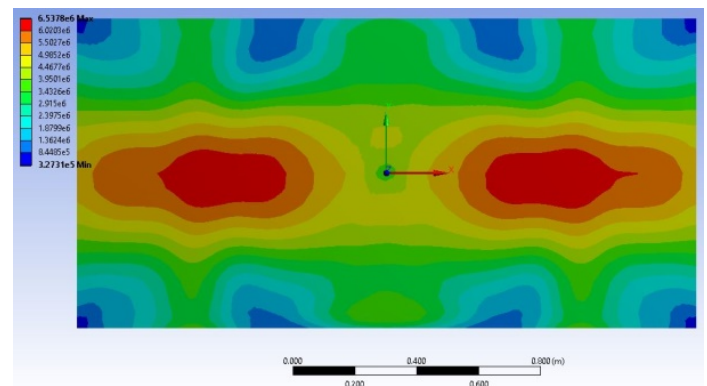

Figure 26 Equivalent stress (Von Mises) distribution diagram for step 22

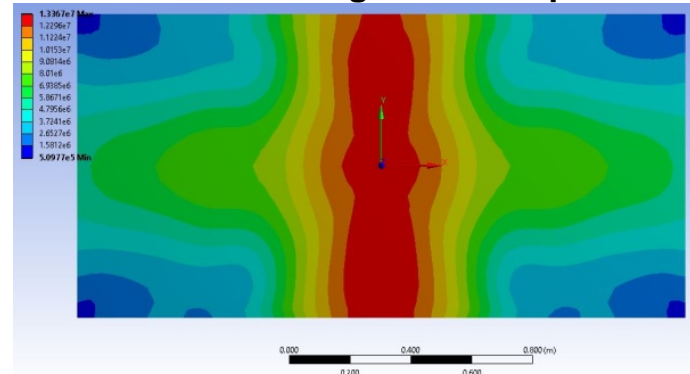

Figure 27 Equivalent stress (Von Mises) distribution diagram for step 23

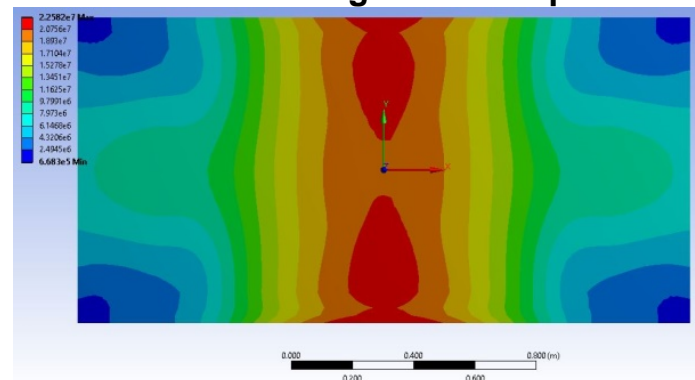

Figure 28 Equivalent stress (Von Mises) distribution diagram for step 24

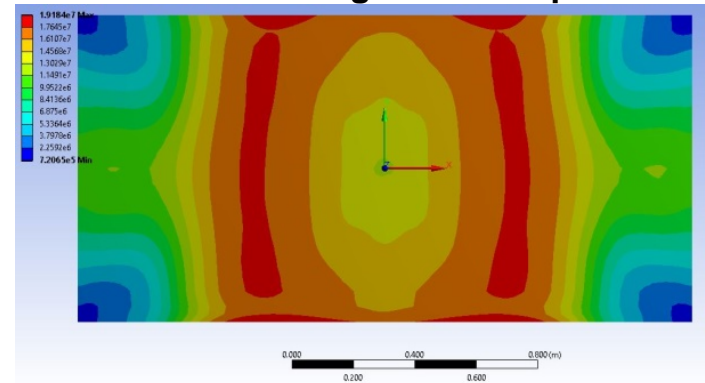

Figure 29 Equivalent stress (Von Mises) distribution diagram for step 25

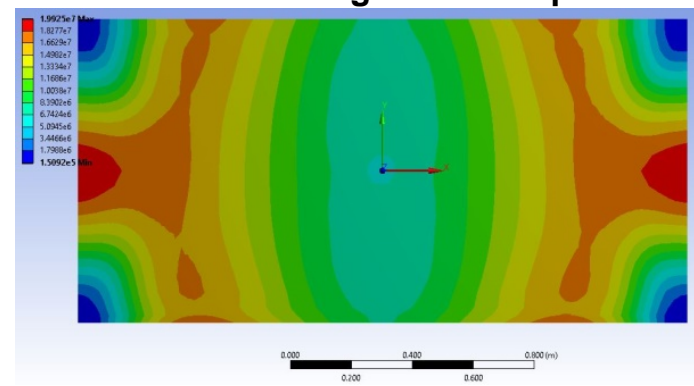

Figure 30 Equivalent stress (Von Mises) distribution diagram for step 26

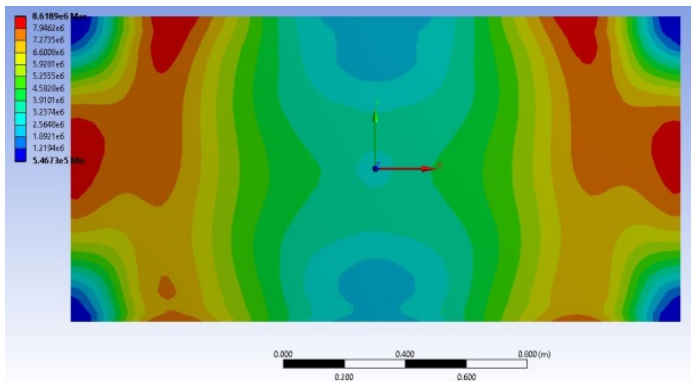

Figure 31 Equivalent stress (Von Mises) distribution diagram for step 27

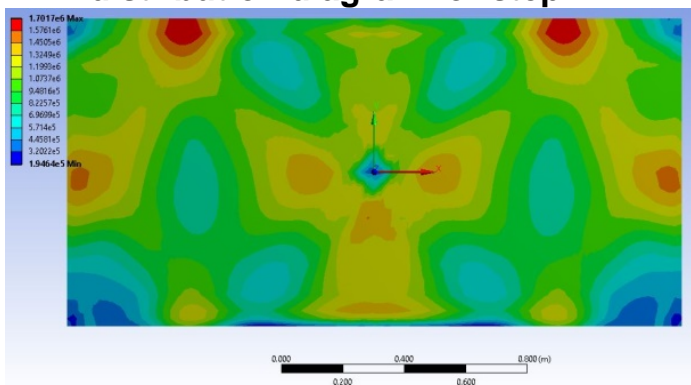

Figure 32 Equivalent stress (Von Mises) distribution diagram for step 28

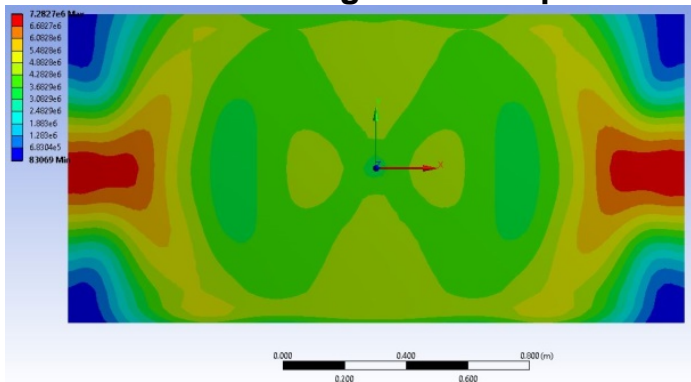

Figure 33 Equivalent stress (Von Mises) distribution diagram for step 29

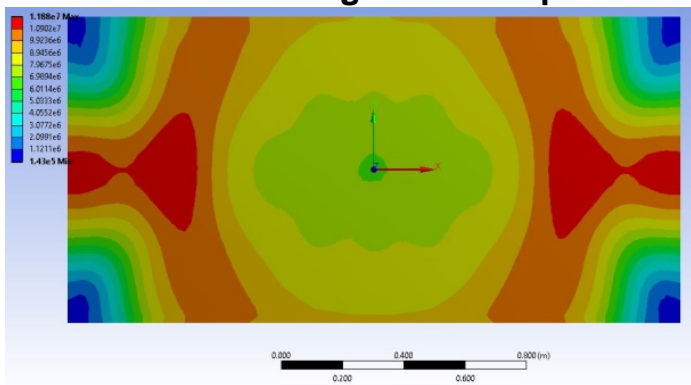

Figure 34 Equivalent stress (Von Mises) distribution diagram for step $\mathbf{3 0}$ 
"Mircea cel Batran" Naval Academy Scientific Bulletin, Volume XIX - 2016 - Issue 2 The journal is indexed in: PROQUEST I DOAJ / Crossref / EBSCOhost / INDEX COPERNICUS / DRJI / OAJI I JOURNAL INDEX I I2OR / SCIENCE LIBRARY INDEX / Google Scholar / Academic Keys/ ROAD Open Access I Academic Resources / Scientific Indexing Services / SCIPIO / JIFACTOR

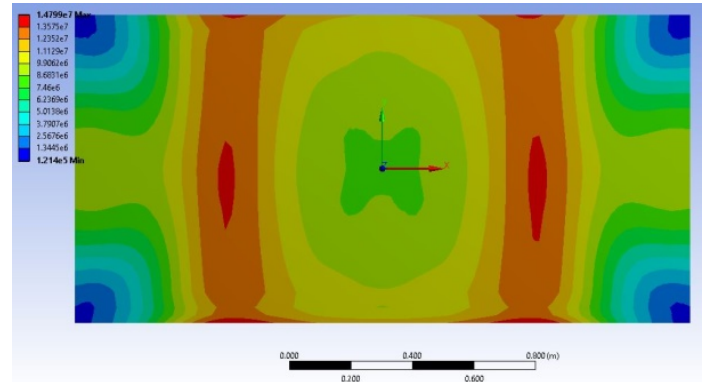

Figure 35 Equivalent stress (Von Mises) distribution diagram for step 31

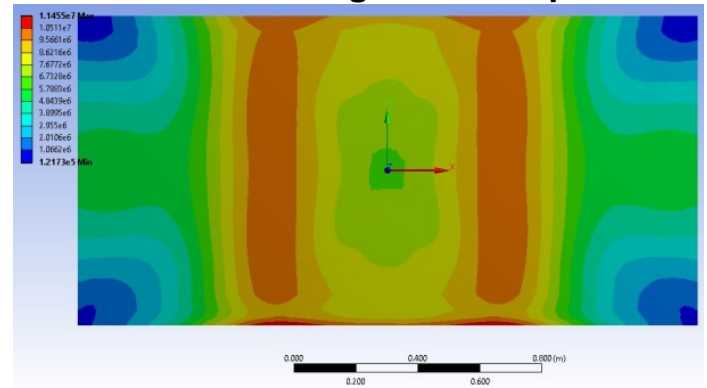

Figure 36 Equivalent stress (Von Mises) distribution diagram for step 32

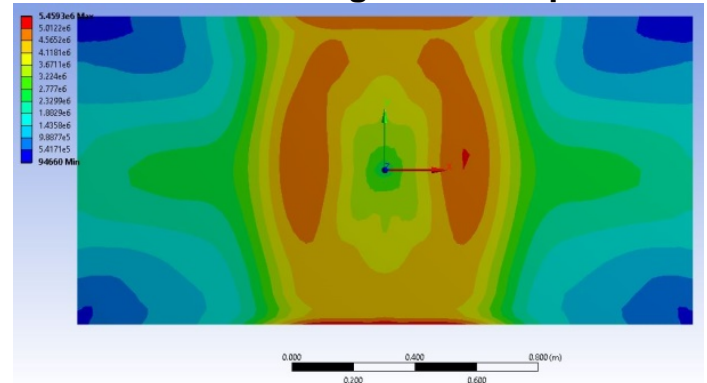

Figure 37 Equivalent stress (Von Mises) distribution diagram for step 33

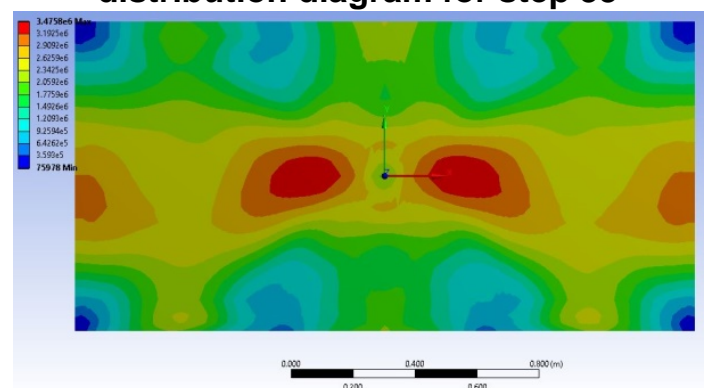

Figure 38 Equivalent stress (Von Mises) distribution diagram for step 34

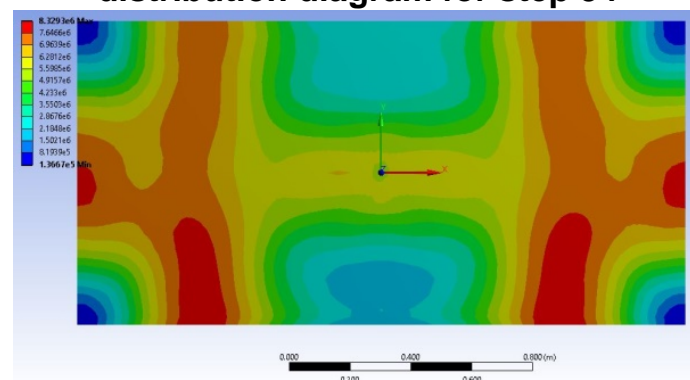

Figure 39 Equivalent stress (Von Mises) distribution diagram for step 35

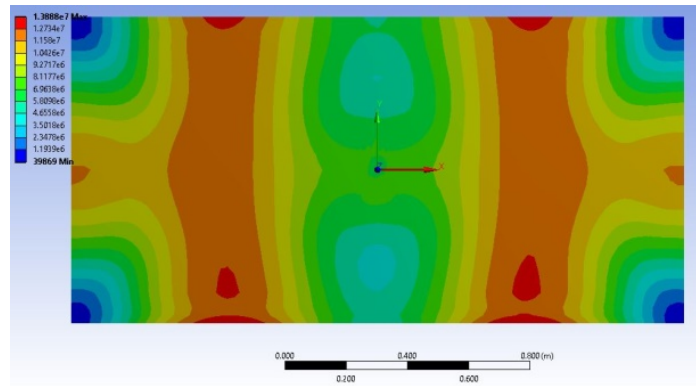

Figure 40 Equivalent stress (Von Mises) distribution diagram for step 36

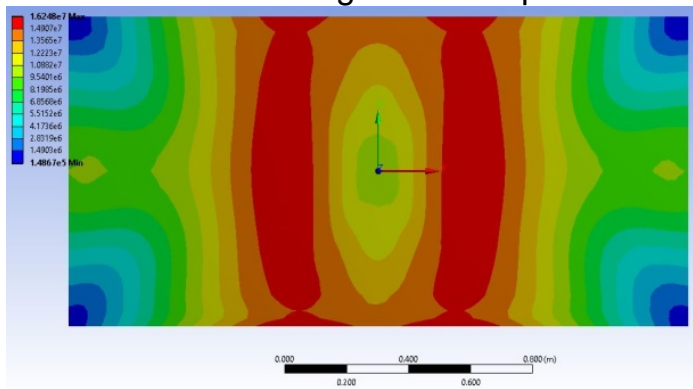

Figure 41 Equivalent stress (Von Mises) distribution diagram for step 37

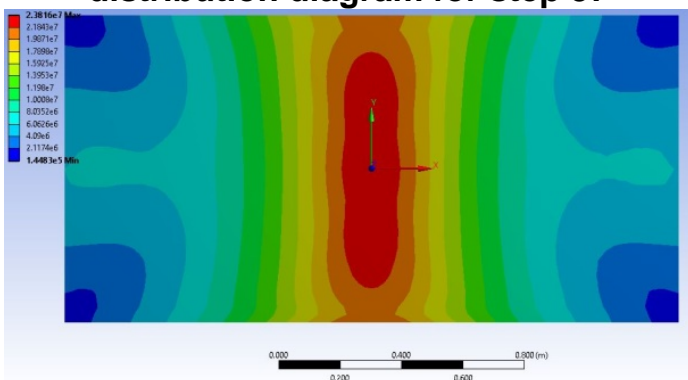

Figure 42 Equivalent stress (Von Mises) distribution diagram for step 38

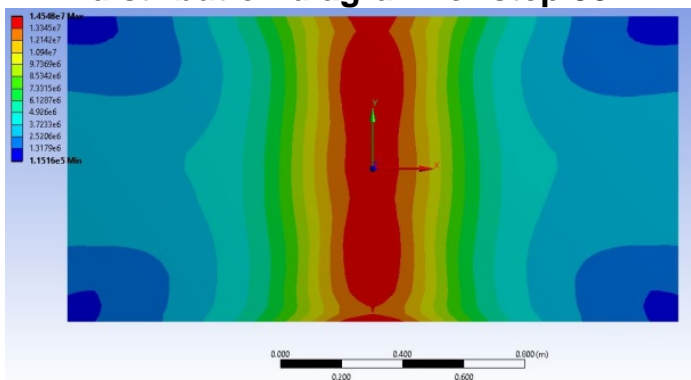

Figure 43 Equivalent stress (Von Mises) distribution diagram for step 39

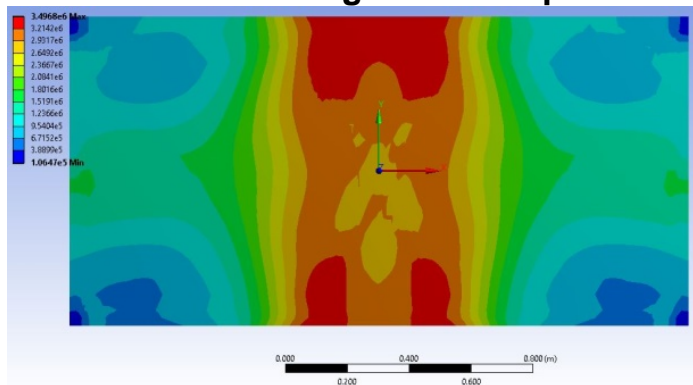

Figure 44 Equivalent stress (Von Mises) distribution diagram for step $\mathbf{4 0}$ 
"Mircea cel Batran" Naval Academy Scientific Bulletin, Volume XIX - 2016 - Issue 2

The journal is indexed in: PROQUEST I DOAJ / Crossref / EBSCOhost / INDEX COPERNICUS / DRJI / OAJI I JOURNAL INDEX I I2OR / SCIENCE LIBRARY INDEX / Google Scholar / Academic Keys/ ROAD Open Access I Academic Resources / Scientific Indexing Services / SCIPIO / JIFACTOR

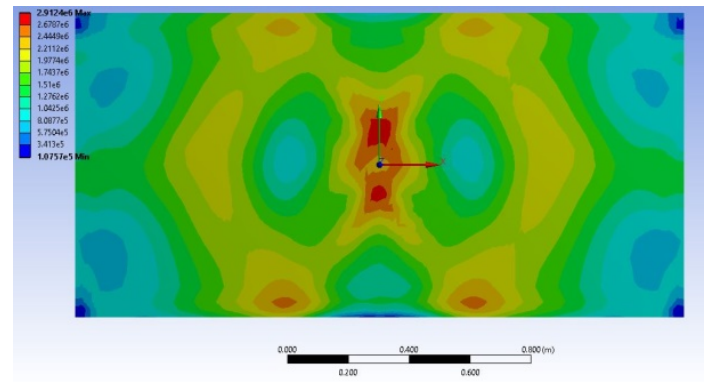

Figure 45 Equivalent stress (Von Mises) distribution diagram for step 41

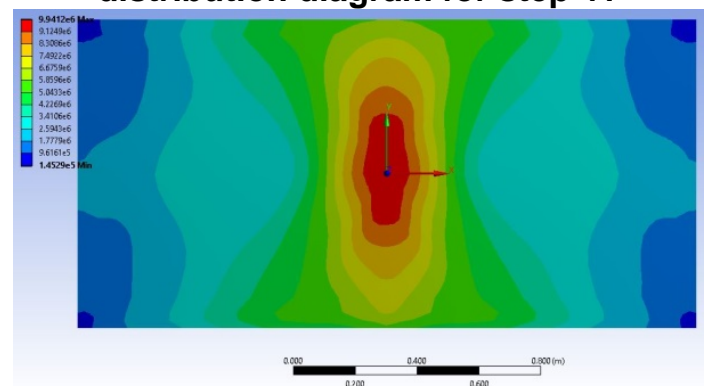

Figure 46 Equivalent stress (Von Mises) distribution diagram for step 42

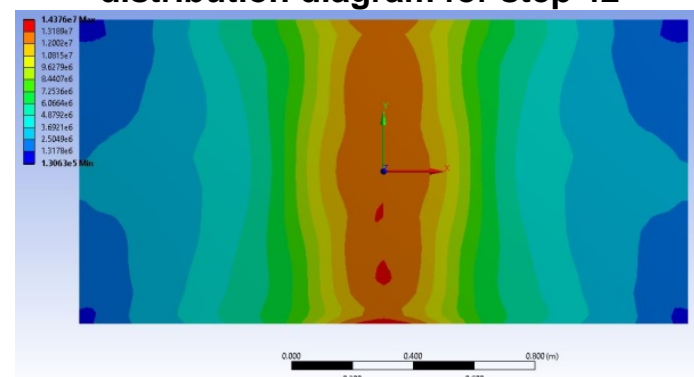

Figure 47 Equivalent stress (Von Mises) distribution diagram for step 43

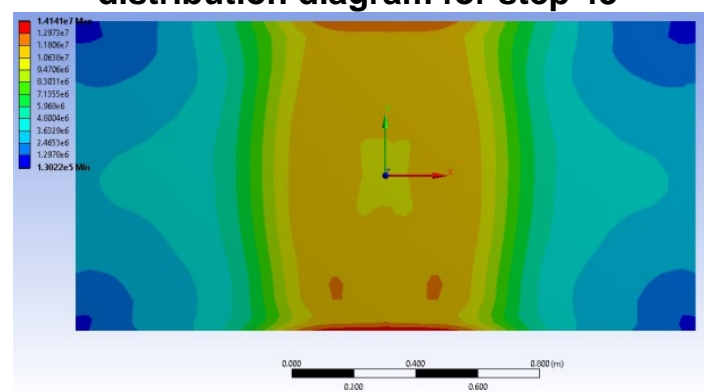

Figure 48 Equivalent stress (Von Mises) distribution diagram for step 44

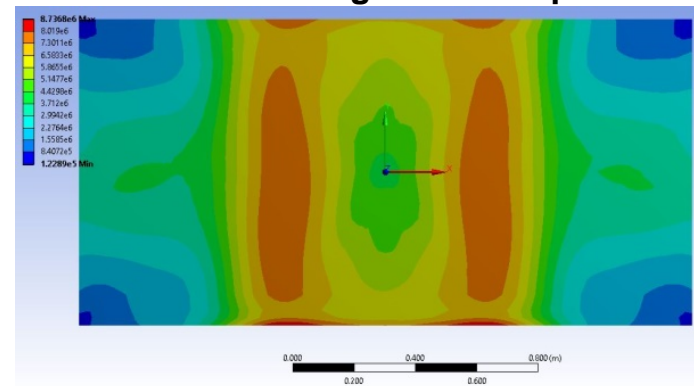

Figure 49 Equivalent stress (Von Mises) distribution diagram for step 45

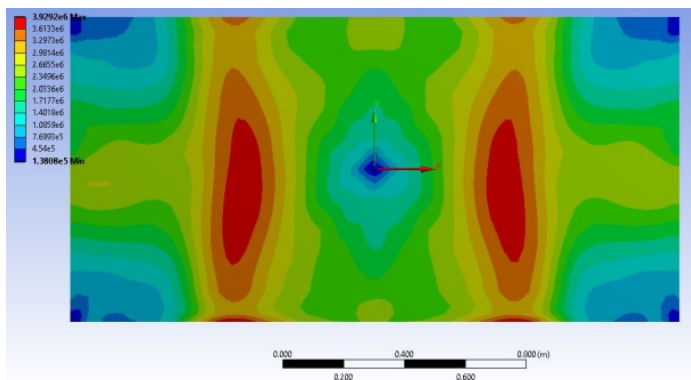

Figure 50 Equivalent stress (Von Mises) distribution diagram for step 46

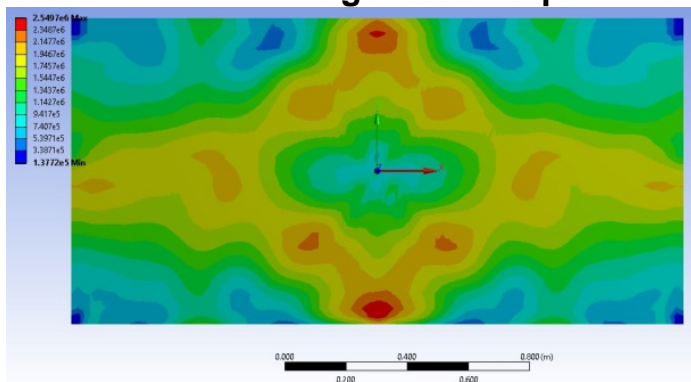

Figure 51 Equivalent stress (Von Mises) distribution diagram for step 47

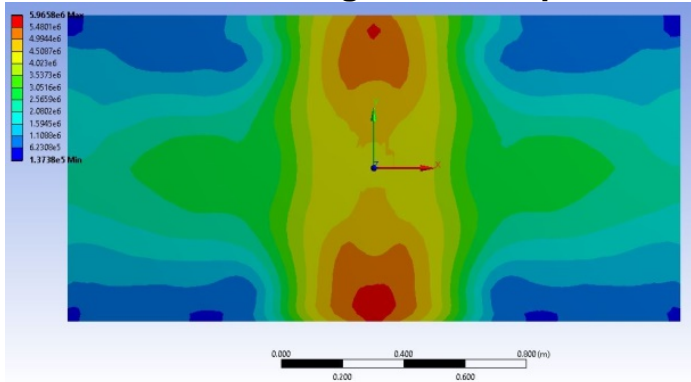

Figure 52 Equivalent stress (Von Mises) distribution diagram for step 48

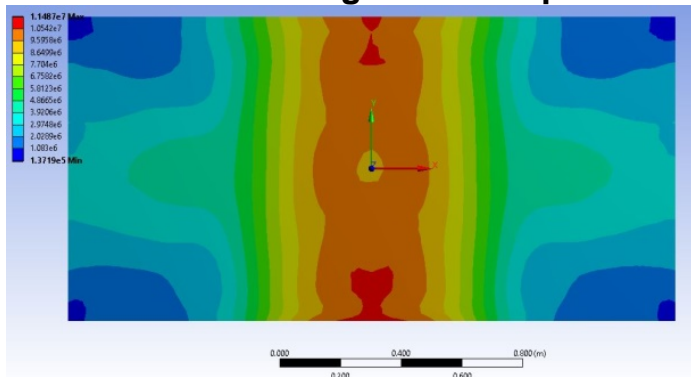

Figure 53 Equivalent stress (Von Mises) distribution diagram for step 49

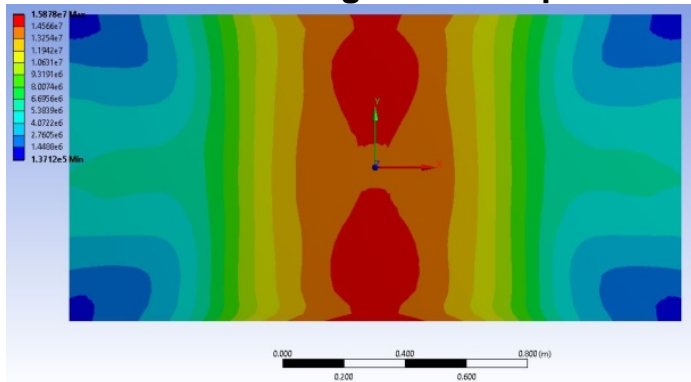

Figure 54 Equivalent stress (Von Mises) distribution diagram for step $\mathbf{5 0}$ 
"Mircea cel Batran" Naval Academy Scientific Bulletin, Volume XIX - 2016 - Issue 2 The journal is indexed in: PROQUEST I DOAJ / Crossref / EBSCOhost / INDEX COPERNICUS / DRJI / OAJI I JOURNAL INDEX I I2OR / SCIENCE LIBRARY INDEX / Google Scholar / Academic Keys/ ROAD Open Access I Academic Resources / Scientific Indexing Services / SCIPIO / JIFACTOR

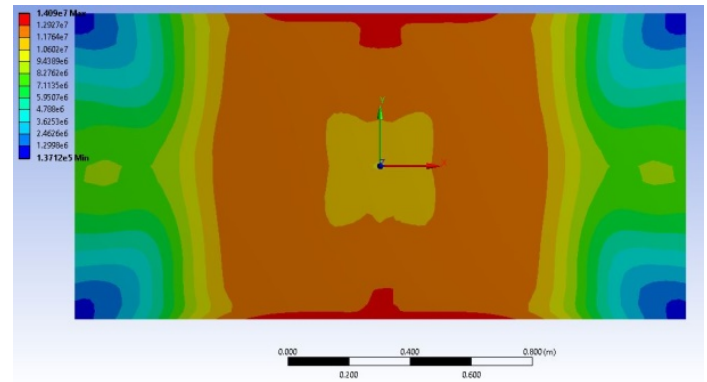

Figure 55 Equivalent stress (Von Mises) distribution diagram for step 51

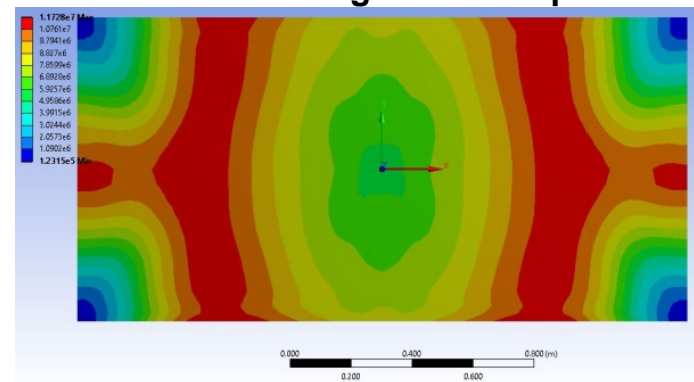

Figure 56 Equivalent stress (Von Mises) distribution diagram for step 52

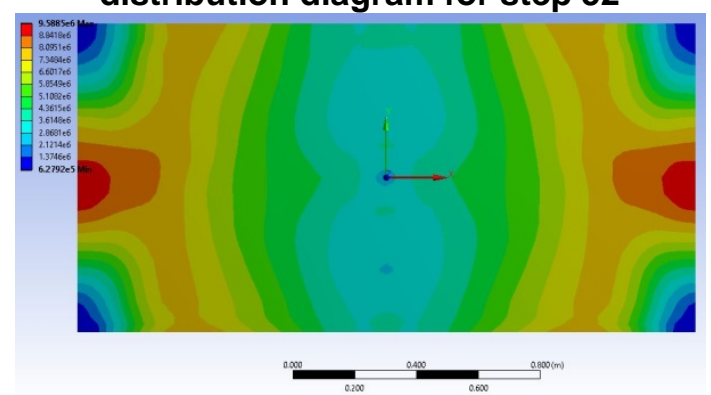

Figure 57 Equivalent stress (Von Mises) distribution diagram for step $\mathbf{5 3}$

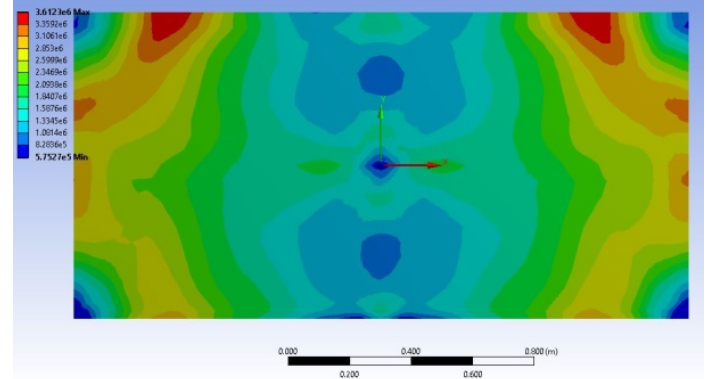

Figure 58 Equivalent stress (Von Mises) distribution diagram for step 54

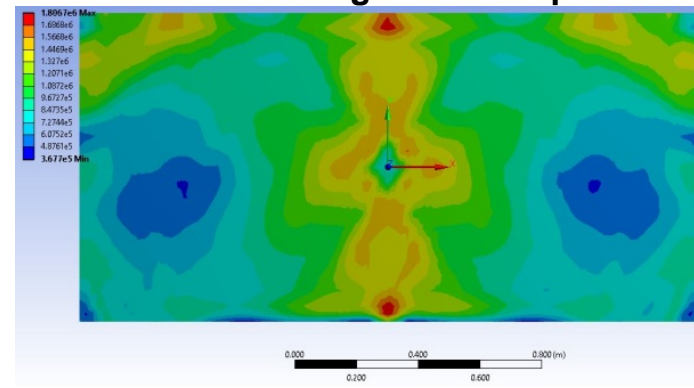

Figure 59 Equivalent stress (Von Mises) distribution diagram for step 55

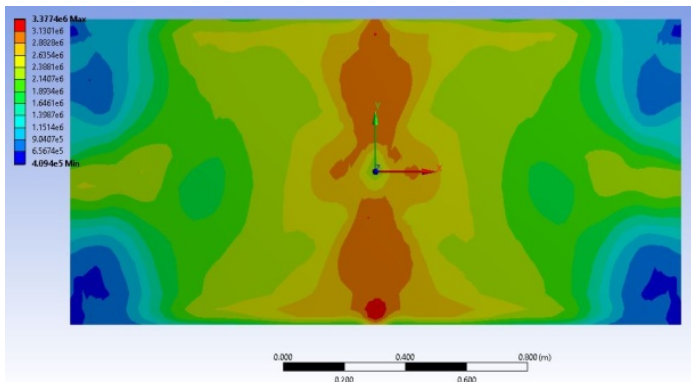

Figure 60 Equivalent stress (Von Mises) distribution diagram for step 56

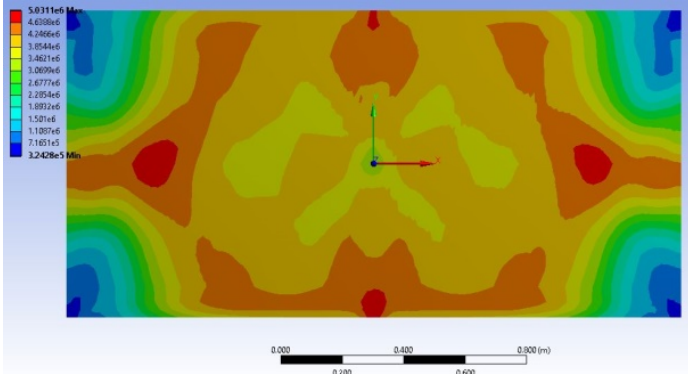

Figure 61 Equivalent stress (Von Mises) distribution diagram for step $\mathbf{5 7}$

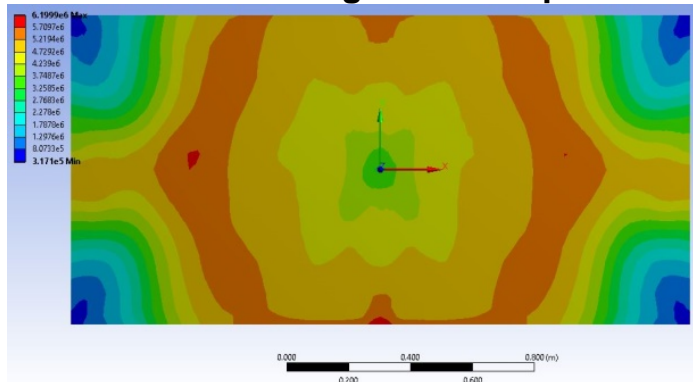

Figure 62 Equivalent stress (Von Mises) distribution diagram for step 58

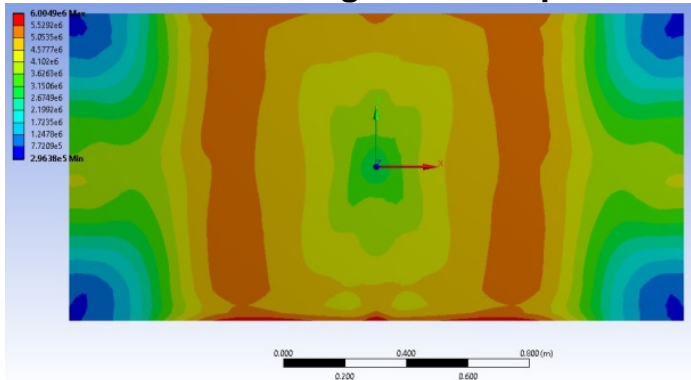

Figure 63 Equivalent stress (Von Mises) distribution diagram for step 59

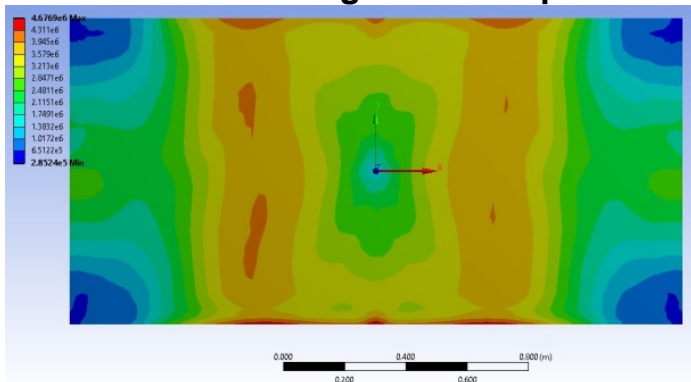

Figure 64 Equivalent stress (Von Mises) distribution diagram for step 60 
"Mircea cel Batran" Naval Academy Scientific Bulletin, Volume XIX - 2016 - Issue 2

The journal is indexed in: PROQUEST I DOAJ / Crossref / EBSCOhost / INDEX COPERNICUS / DRJI / OAJI I JOURNAL INDEX I I2OR / SCIENCE LIBRARY INDEX / Google Scholar / Academic Keys/ ROAD Open Access I Academic Resources / Scientific Indexing Services / SCIPIO / JIFACTOR

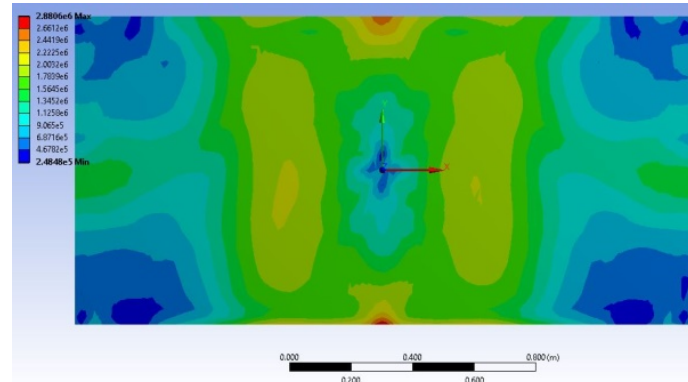

Figure 65 Equivalent stress (Von Mises) distribution diagram for step 61
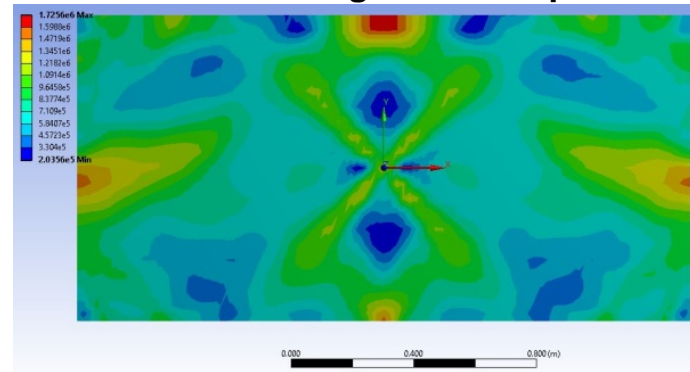

Figure 66 Equivalent stress (Von Mises) distribution diagram for step 62

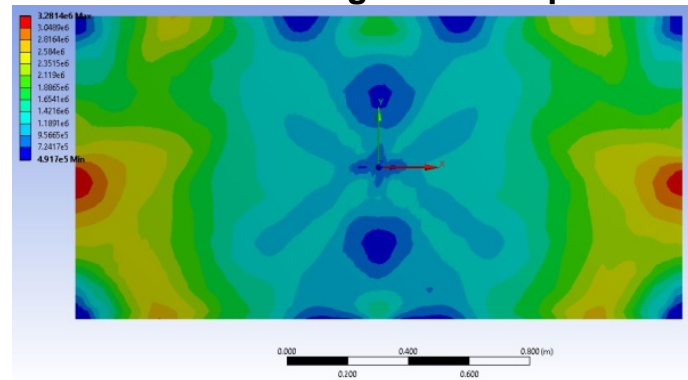

Figure 67 Equivalent stress (Von Mises) distribution diagram for step 63

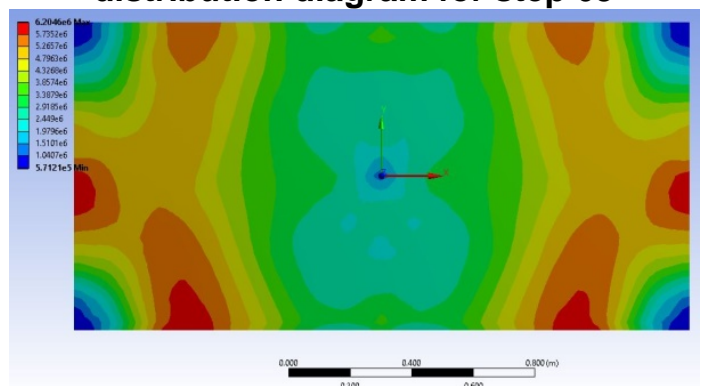

Figure 68 Equivalent stress (Von Mises) distribution diagram for step 64

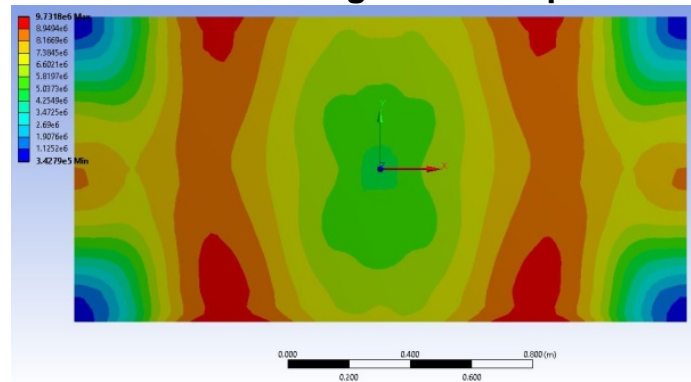

Figure 69 Equivalent stress (Von Mises) distribution diagram for step 65

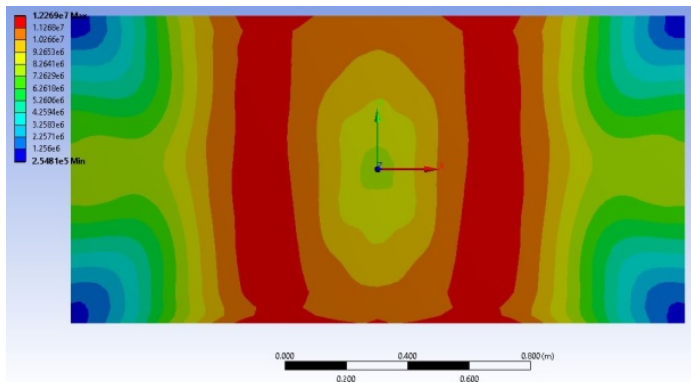

Figure 70 Equivalent stress (Von Mises) distribution diagram for step 66

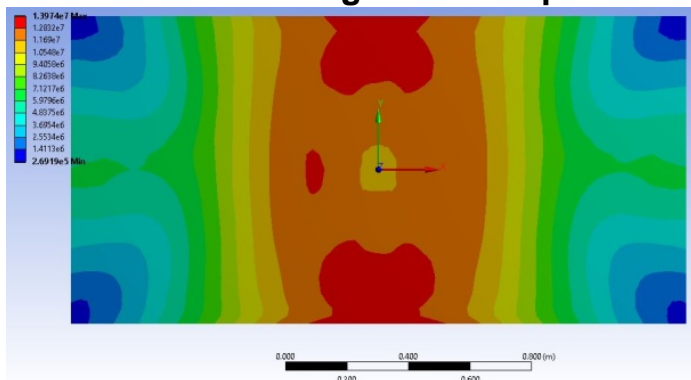

Figure 71 Equivalent stress (Von Mises) distribution diagram for step 67

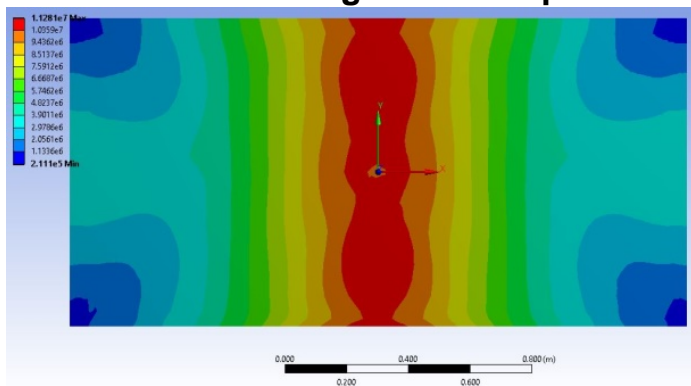

Figure 72 Equivalent stress (Von Mises) distribution diagram for step 68

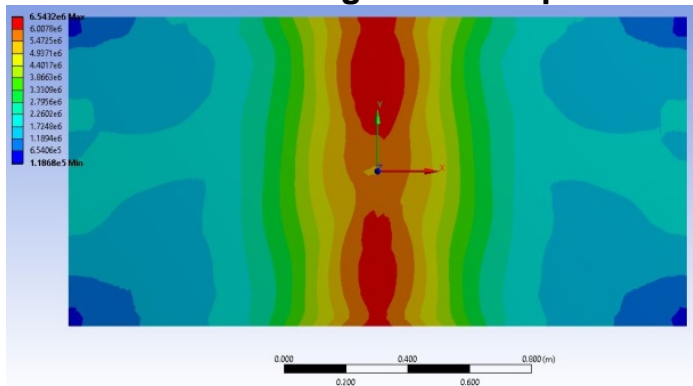

Figure 73 Equivalent stress (Von Mises) distribution diagram for step 69

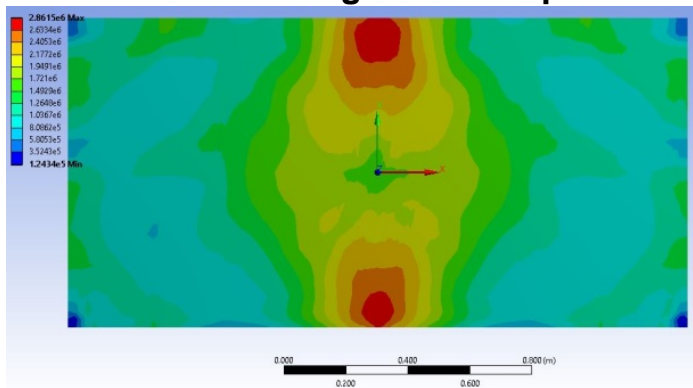

Figure 74 Equivalent stress (Von Mises) distribution diagram for step 70 
"Mircea cel Batran" Naval Academy Scientific Bulletin, Volume XIX - 2016 - Issue 2

The journal is indexed in: PROQUEST I DOAJ / Crossref / EBSCOhost / INDEX COPERNICUS / DRJI / OAJI I JOURNAL INDEX / I2OR / SCIENCE LIBRARY INDEX / Google Scholar / Academic Keys/ ROAD Open Access I Academic Resources / Scientific Indexing Services / SCIPIO / JIFACTOR

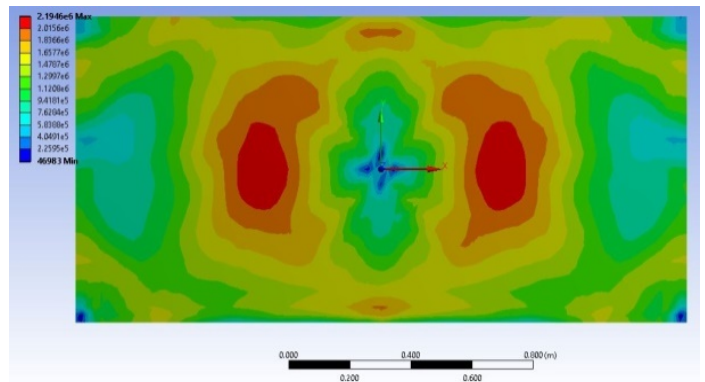

Figure 75 Equivalent stress (Von Mises) distribution diagram for step 71

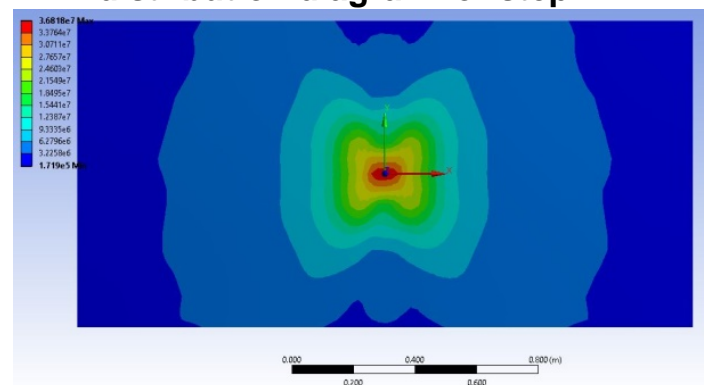

Figure 76 Equivalent stress (Von Mises) distribution diagram for step 72

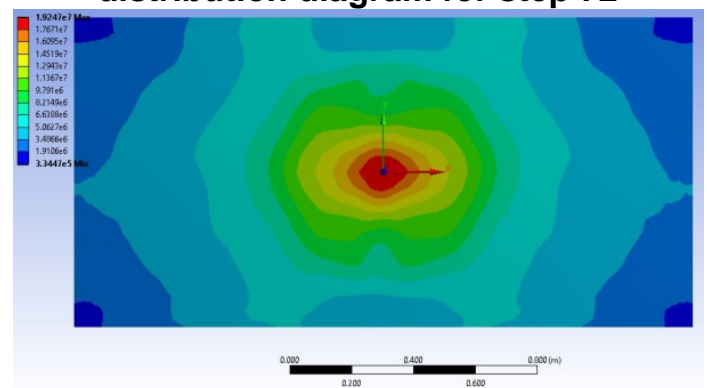

Figure 77 Equivalent stress (Von Mises) distribution diagram for step 73

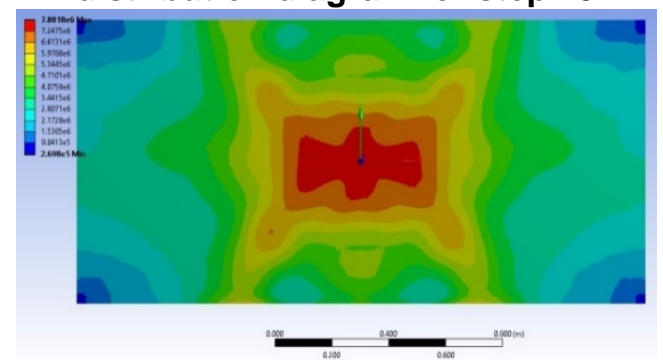

Figure 78 Equivalent stress (Von Mises) distribution diagram for step $\mathbf{7 4}$

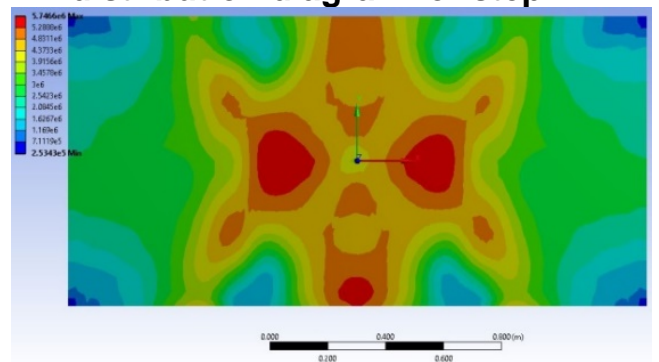

Figure 79 Equivalent stress (Von Mises) distribution diagram for step 75

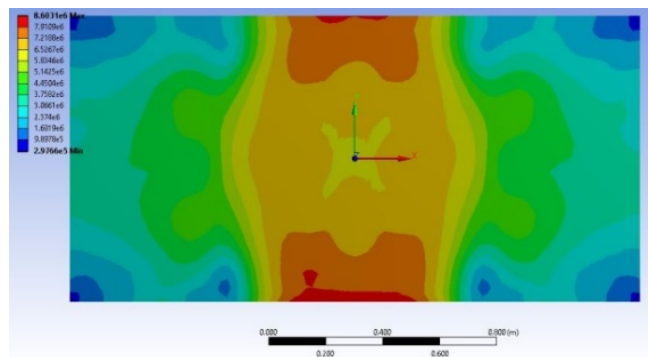

Figure 80 Equivalent stress (Von Mises) distribution diagram for step 76

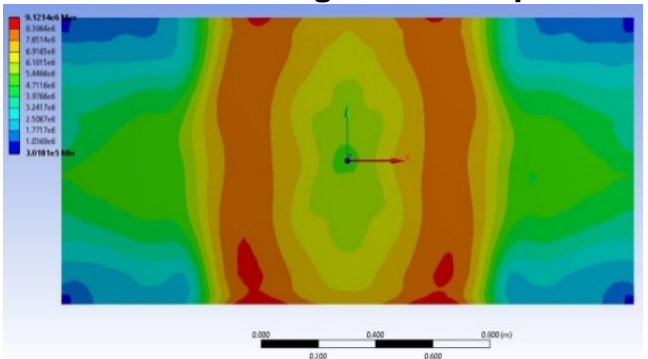

Figure 81 Equivalent stress (Von Mises) distribution diagram for step 77

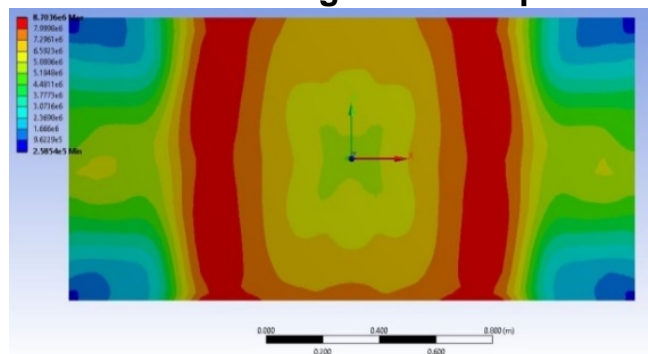

Figure 82 Equivalent stress (Von Mises) distribution diagram for step 78

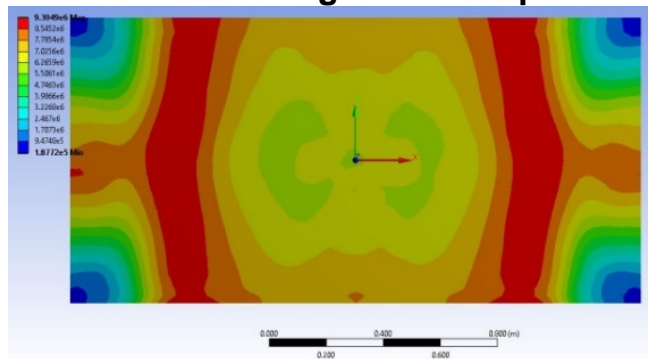

Figure 83 Equivalent stress (Von Mises) distribution diagram for step $\mathbf{7 9}$

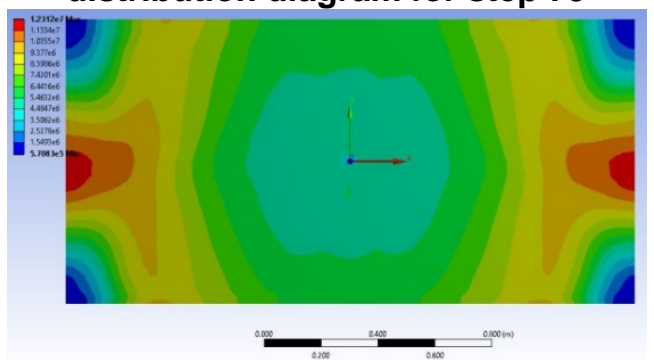

Figure 84 Equivalent stress (Von Mises) distribution diagram for step $\mathbf{8 0}$ 
"Mircea cel Batran" Naval Academy Scientific Bulletin, Volume XIX - 2016 - Issue 2 The journal is indexed in: PROQUEST / DOAJ / Crossref / EBSCOhost / INDEX COPERNICUS / DRJI / OAJI I JOURNAL INDEX I I2OR / SCIENCE LIBRARY INDEX / Google Scholar / Academic Keys/ ROAD Open Access I Academic Resources / Scientific Indexing Services / SCIPIO / JIFACTOR

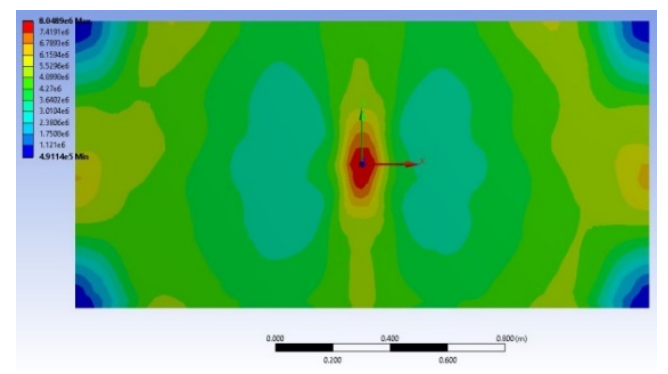

Figure 85 Equivalent stress (Von Mises) distribution diagram for step 81

\section{CONCLUSIONS}

The maximum value of von Mises tensions are reach at step $3(7.5 \mathrm{~ms})$ with a value of $8.99 \mathrm{E} 7 \mathrm{~N} / \mathrm{m}^{2}$ $\left(89.9 \mathrm{~N} / \mathrm{mm}^{2}\right)$.

\section{BIBLIOGRAPHY}

[1] Ansys Workbench User Manual

[2] Huei - Huang Lee, Finite Element Simulations with Ansys Workbench 12, Schroff Development Corporation, ISBN 978-1-58503-604-2, 2010,

[3] Moaveni Saeed, Finite Element Analysis: Theory and applications with Ansys, 3rd edition, ISBN978-0-13189080-0, 2008

[4] O.C. Zienkiewicz, R.L. Taylor, The Finite Element Method for Solid and Structural Mechanics, 6th Edition, ISBN 0-7506-6321-9, 2005 